\title{
Inverse filtering for time, delay and integration X-ray imaging
}

\author{
M. Rosenbaum, W. Sauer-Greff, and R. Urbansky \\ TU Kaiserslautern, Institute of Communications Engineering, 67653 Kaiserslautern, Germany
}

\begin{abstract}
In food industry, most finished products are scanned by X-ray for contaminations. These X-ray machines continuously scan the product passing through. To minimize the required X-ray power, a Time, Delay and Integration (TDI) CCD sensor is used to capture the image. While the product moves across the sensor area, the angle of the $\mathrm{X}$-rays changes during the pass. This can be compensated for by adjusting the sensor shift speed to focus on a single plane of the product. If the product has a significant thickness, the image will show artifacts due to the laminographic effect. In this contribution we demonstrate that by the use of inverse filtering images which are focused on planes of different height can be generated out of a single X-ray image.
\end{abstract}

\section{Introduction}

In food industry, there is a need to prevent contaminated products from leaving the production site. Metal detectors are widely used for this task, but they fail for non-metallic objects. Glass splinters and ceramic objects such as broken ceramic knife blades or ceramic ball bearing balls can not be detected.

Continous X-ray imaging allows to detect all these objects and the machine can simply be fitted in the production process. The product passes the X-ray machine continously on a belt with an overhead X-ray source. After passing the product, the X-rays are converted to visible light by a szintillating foil. The light emitted by this foil is projected on a lineaccumulating CCD sensor. A typical sensor has 128 lines which are shifted synchronously to the transport belt and the charges are moved from line to line over the sensor accumulating more and more X-ray light. Each image leaving the sensor is exposed 128 times. This allows for a high efficiency and low noise, however the angle of the X-ray beam passing through the product changes slightly from shift to shift. This produces a so-called laminographic effect. By

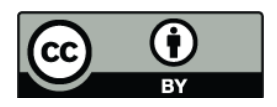

Correspondence to: M. Rosenbaum (rosenbaum@eit.uni-kl.de) this effect, object edges get washed out, the sharpness of the image is reduced in general. Because of the imaging process, it is not possible to distinguish between a flat, dense object and a high, less dense object - e.g. metallic washer ring versus macaroni noodle. Figure 1 shows a schematic drawing of such an X-ray scanner.

Currently, it is necessary to use laminographic techniques (Gondrom, 1999; Rooks, 1995) in conjunction with the Radon Transform (Popularikas, 1996) if depth information is needed. This requires two X-ray sources and two sensor assemblies which deliver images of the product under different angles. This is not only expensive, in addition the second $\mathrm{X}$-ray tube also needs cooling and better shielding against $\mathrm{X}$-rays leaving the machine is required. In this paper, we propose the use inverse filtering of the single X-ray sensor image to calculate images focussed on arbitrary planes of the product. The paper is organized as follows: first, we give an short introduction into the X-ray imaging process. After that, the inverse filtering is discussed, followed by the description of the test setup and the results.

\section{Imaging process}

The product is transported through the X-ray scanner on a conveyor belt. Above the belt, an X-ray source is located, the imaging equipment can be found below the belt. Because large CCD sensors are very expensive and subject to damage by $\mathrm{X}$-ray radiation, a szintillation foil which converts X-rays into visible light is located below the conveyor belt. The light is focussed by optic lenses and digitalised by a CCD sensor. This CCD sensor has a typical resolution of $128 \times 1024$ pixel. The electrical charge generated by photons hitting the sensor is shifted from line to line and read out after it reaches the last line (in this case line 128). Each line which enters the analsysis system has been exposed 128 times, which can be modeled as a superposition of 128 complete images. Figure 1 shows a schematical drawing of such an apparatus.

The angle under which the $\mathrm{X}$-rays enter the product changes as the belt moves the product across the sensor. Therefor the image gets blurred in movement direction. This

Published by Copernicus Publications on behalf of the URSI Landesausschuss in der Bundesrepublik Deutschland e.V. 


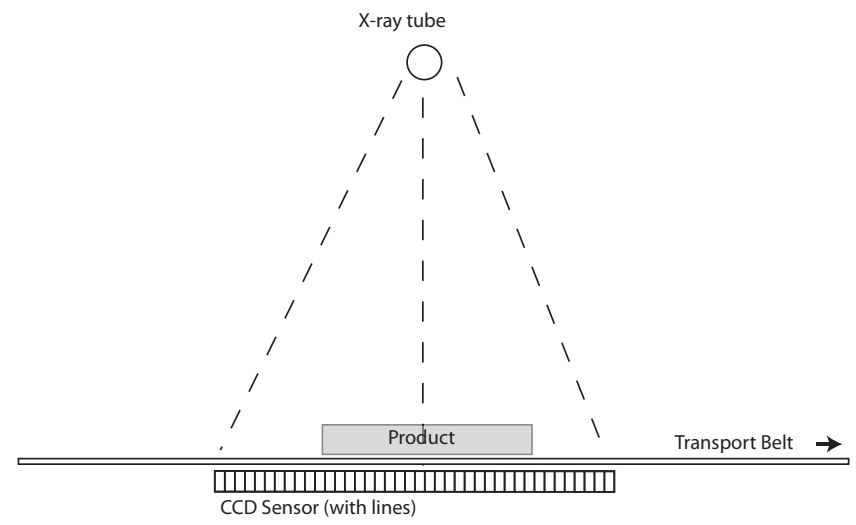

Fig. 1. Schematic drawing of the X-ray scanner.

effect makes it difficult to reliably detect edges. For one specifice height, this can be compensated by an offset between belt- and sensor-shift-speed. If two sharp planes are needed, two scans have to be performed. Also, there is no depth information in the resulting image and no differences between flat and non-flat objects are visible.

Because of the width of the optical active area, the object is X-rayed at different angles. The charge shift can be modeled as the superposition of 128 separate images taken under different angles. How large this angle is depends on the specific scanner setup, but in general an angle below 5 degrees is used. This superposition of images under different angles leads to blurring, the so-called laminographic effect.

This effect can be modeled as a convolution of the real image $a(x, y)$ with the point spread function of the imaging system $h(x, y)$, yielding an image $s(x, y)$.

$s(x, y)=a(x, y) \cdot h(x, y)$

In general, this function is two-dimensional, but in our case there is a significant contribution only in direction of the belt movement, which can be modeled as a one-dimensional impulse response. This impulse response is height variant, so it is only valid for a given height of the object above the sensor plane. This means that only flat objects can be diplayed sharp. If the object is non-flat, only one slice would be diplayed sharp, and the parts of the object out of focus produce blurring.

Figure 2 depicts the calculated impulse response for a distance of $8 \mathrm{~cm}$ between object and sensor plane. This impulse response can be calculated from the X-ray image of a well known object.

\section{Inverse filtering}

This blurring caused by the laminographic effect can be effectively addressed by inverse filtering since it can be regarded as a convolution of the real image with the

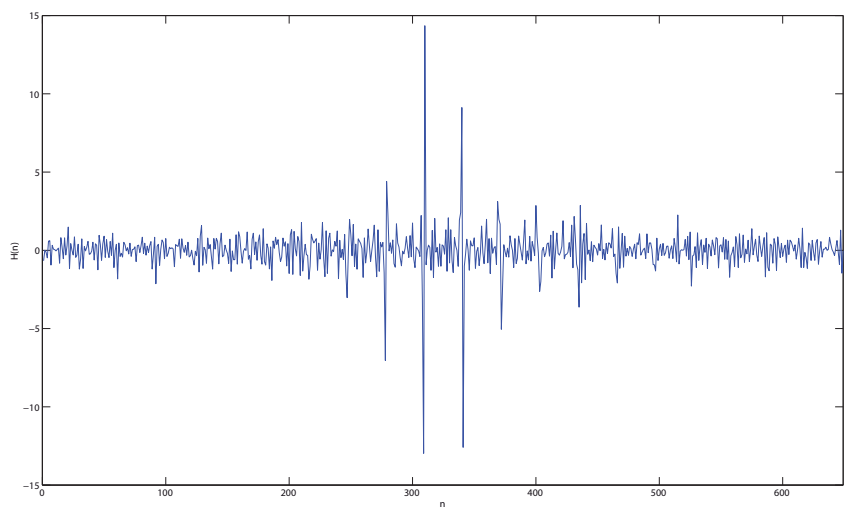

Fig. 2. Calculated impulse response.

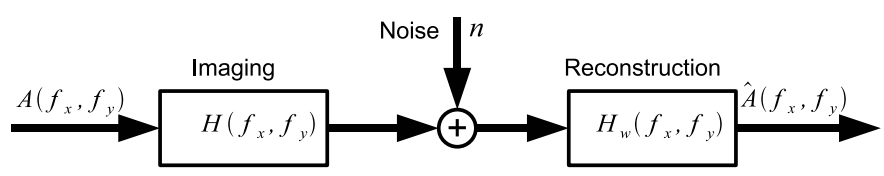

Fig. 3. Block diagram.

point-spread-function of the imaging system corrupted with noise $n$.

$s(x, y)=a(x, y) \cdot h(x, y)+n$

The inverse filtering takes advantage of the frequency domain. A pure inversion of the transfer function $H\left(f_{\mathrm{x}}, f_{\mathrm{y}}\right)$ requires to take the reciprocal of it yielding $H_{\mathrm{w}}\left(f_{\mathrm{x}}, f_{\mathrm{y}}\right)=$ $H^{-1}\left(f_{\mathrm{x}}, f_{\mathrm{y}}\right)$, but this poses new problems because of spectral zeros and noise contained in the X-ray image will be amplified. To deal with this, Wiener filtering (Madisetti, 1998) can be used. Equation (3) shows the parametric form of the Wiener filter. $K$ accounts for noise influence, ideally it is choosen as the spectral power density of the white noise contained in the X-ray image.

$H_{\mathrm{w}}\left(f_{\mathrm{x}}, f_{\mathrm{y}}\right)=\frac{H^{*}\left(f_{\mathrm{x}}, f_{\mathrm{y}}\right)}{\left|H\left(f_{\mathrm{x}}, f_{\mathrm{y}}\right)\right|^{2}+K}$

Figure 3 shows the imaging and reconstruction process, producing an estimate $\hat{A}$ of the unknown original image $A$.

In the reconstruction process, an impulse response is only valid for a single focal plane in the X-ray image. The characteristics of the imaging process allow to calculate an impulse response for every focal plan out of one known impulse response by using algorithms also known in image processing as bilinear or bicubic scaling (Keys, 1981). To generate a depth map of the objects, overlapping focal planes need to be calculated and tested if an object is sharp in one focal plane. If this is the case, the object is flat and its height is known. If the object is non-flat, it is not sharp in any focal plane. 


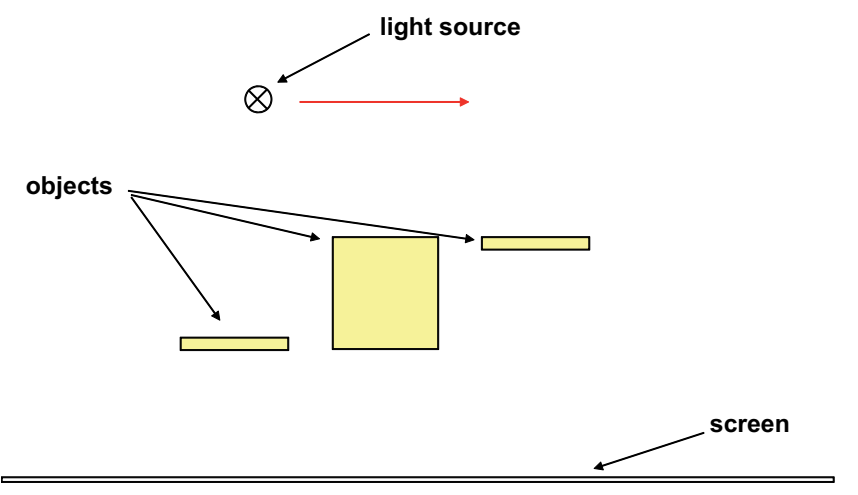

Fig. 4. Simulation setup.
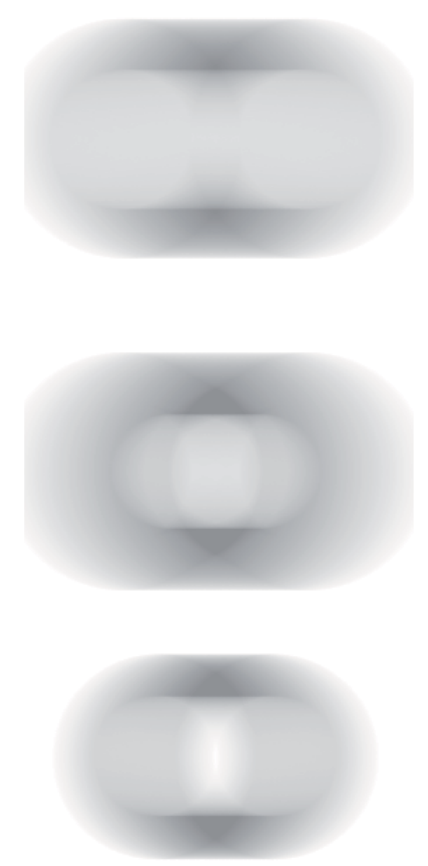

Fig. 5. Simulated X-ray image.

\section{Simulation setup}

To demonstrate the proposed strategy, test images were generated using a ray-tracing software. The test setup contains two thin rings at different distances to the screen and a long tube with the same diameter as the rings. 128 images were rendered and overlayed to produce the final image. This image was used to test the inverse filtering strategy. Figure 4 shows schematically the setup used for the simulations. The impulse response was estimated by geometrical calculations, $K$ in Eq. (3) was choosen to $10^{-4}$.

Figure 5 depicts a blurred image of the 3 objects which results after adding up all 128 rendered images.
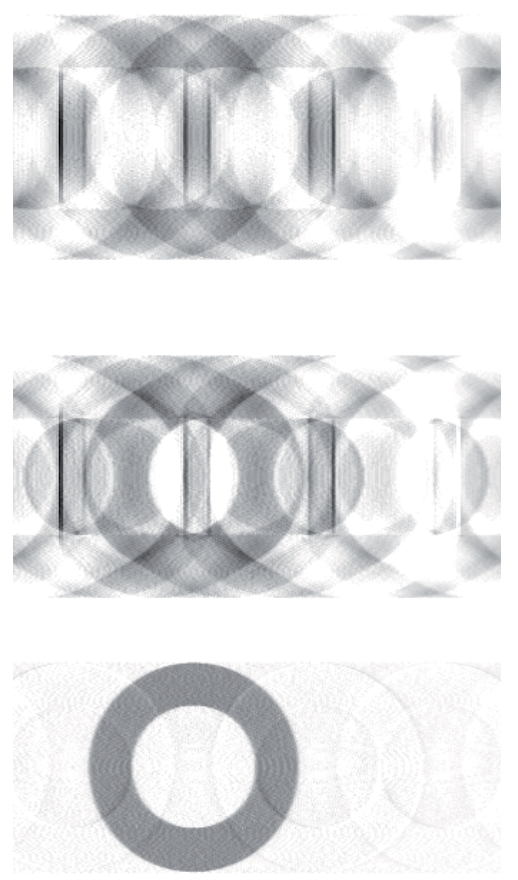

Fig. 6. Focus on lower ring.
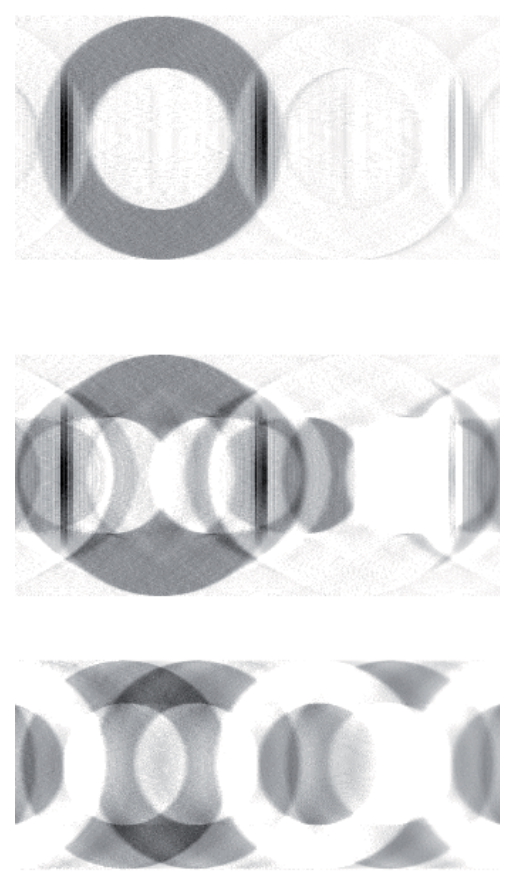

Fig. 7. Focus on upper ring.

\section{Results}

The generated source image was inversely filtered with an estimated impulse response as shown in Fig. 2. The first image (Fig. 6) shows the result if focussed to the lower ring. The upper ring and the cylinder are distorted by a lot of artifacts. 
As opposed to Fig. 6, Fig. 7 shows the result if focussed to the upper ring. This was done by scaling the impulse response used before. As expected, flat objects can be focussed, high objects are not sharp in any focal plane. The slight artifacts visible even in the sharp rings result from inaccuracies in the estimation procedure of the impulse response.

\section{Conclusions}

The presented technique allows it to gain depth information out of a single image corrupted by the laminographic effect. This allows a classification of targets into flat and non-flat objects and also to calculate the height of a flat object above the conveyor belt. This technique is easy to implement as it works with already existing sensors and machinery, only the analysis software needs to be modified. This allows the subsequent classification algorithms a more reliable decision whether an object belongs to the product or is a contamination.

\section{References}

Gondrom, S. and Schröpfer, S.: Digital computed laminography and tomosynthesis - functional principles and industrial applications, Proceedings of International Symposium on Computed Tomography and Application, Berlin, Germany, 1999.

Keys, R.: Cubic convolution interpolation for digital image processing, IEEE Transactions on Signal Processing, Acoustics, Speech, and Signal Processing 29, 1981.

Madisetti, V. K. and Williams, D. B. (Eds.): The Digital Signal Processing Handbook, CRC Press, 1998.

Popularikas, A. D. (Ed.): The Transforms and Applications Handbook, CRC Press, 1996.

Rooks, S. and Sack, T.: X-ray Inspection of Flip Chip Attach Using Digital Tomosynthesis, Circuit World, 21(3), 51-55, 1995. 